\title{
Article \\ Electrochemical Deposition of CoP and CoNiP as Hard Magnetic Scales in a Position Measurement System
}

\author{
Martin Theis ${ }^{1,2}$, Tobias Bill ${ }^{3}\left[\right.$, Heiko Knoll ${ }^{1}$, Peter Starke ${ }^{3}\left([)\right.$ and Monika Saumer ${ }^{2, *} \mathbb{C}$ \\ 1 Sensitec GmbH, Heiligkreuzweg 109, D-55130 Mainz, Germany; martin.theis@sensitec.com (M.T.); \\ heiko.knoll@sensitec.com (H.K.) \\ 2 Department of Microsystems and Information Technologies, University of Applied Sciences Kaiserslautern, \\ Amerikastr. 1, D-66482 Zweibruecken, Germany \\ 3 Department of Materials Science and Materials Testing (WWHK), University of Applied Sciences \\ Kaiserslautern, Schoenstraße 11, D-67659 Kaiserslautern, Germany; tobias.bill@hs-kl.de (T.B.); \\ peter.starke@hs-kl.de (P.S.) \\ * Correspondence: monika.saumer@hs-kl.de
}

Citation: Theis, M.; Bill, T.; Knoll, H.; Starke, P.; Saumer, M.

Electrochemical Deposition of CoP and $\mathrm{CoNiP}$ as Hard Magnetic Scales in a Position Measurement System. Metals 2022, 12, 235. https:// doi.org/10.3390/met12020235

Academic Editors: Jannis N. Lemke and Alberto Coda

Received: 21 December 2021

Accepted: 21 January 2022

Published: 26 January 2022

Publisher's Note: MDPI stays neutral with regard to jurisdictional claims in published maps and institutional affiliations.

Copyright: (c) 2022 by the authors. Licensee MDPI, Basel, Switzerland. This article is an open access article distributed under the terms and conditions of the Creative Commons Attribution (CC BY) license (https:/ / creativecommons.org/licenses/by/ $4.0 /)$.

\begin{abstract}
The fabrication and design of hard magnetic materials for micro-electro-mechanical system applications by electrochemical deposition has to consider not only the intrinsic material properties but also the shape anisotropy of the micro-devices. Within the scope of the present work, an asplated process for hard magnetic Co-based materials was developed, with the products intended to be used as magnetic scales in a positioning system with a resolution within the nanometer range. First, the process-material correlations are investigated in a laboratory-scale process. The CoP and CoNiP show a maximum coercivity of $H_{C}=28$ and $45 \mathrm{kA} / \mathrm{m}$, respectively, as well as maximum remanence polarizations of $J_{R}=0.65$ and $0.40 \mathrm{~T}$, respectively. The CoP process is transferred to a specially developed $20 \mathrm{~L}$ plating cell with paddle convection capabilities and a passive bezel to deposit $50 \mu \mathrm{m}$ wide scales with different thicknesses of up to $55 \mu \mathrm{m}$ in an integrated process. The inplane magnetization of the scale bars shows higher remanence polarization than for the out-of-plane direction. Magnetic field-assisted electrochemical deposition promotes the vertical magnetization component resulting in a remanence polarization of $205 \mathrm{mT}$ (out-of-plane) for a scale thickness of $25 \mu \mathrm{m}$.
\end{abstract}

Keywords: process-material correlations; thick hard magnets; electrochemical deposition; integrated process; MEMS

\section{Introduction}

Hard magnetic materials used in magnetic MEMS (micro-electro-mechanical systems) devices require an IC/CMOS compatible fabrication process to achieve an economical edge over the common hard magnetic materials, e.g., sintered $\mathrm{NbFeB}$. Thin-film technologies such as sputtering and molecular beam epitaxy enable the deposition of thin magnetic films, e.g., Co-based or rare earth alloys such as SmCo, with various magnetic anisotropy configurations [1]. However, only relatively thin layers can be provided through these processes, which means that the effective magnetic field strength is usually too low at working distances that are typical for their respective applications. Among the manufacturing processes for thick magnetic layers described in the literature, agglomeration of powder magnets by atomic layer deposition [2] can be used to produce thick, integrated micromagnets, with the drawback that the lower packing density requires the use of rare earth elements to achieve sufficiently high magnetic field strengths at a given distance. Electrochemical deposition of alloys on the other hand, enables the cost-effective production of thick, integrated Co-based micromagnets with a high degree of geometric freedom and precision. A limitation of the application of this process is the small number of materials for which manufacturing processes exist. 
MEMS applications for such micro-magnets are, i.e., as actuators in energy generation and harvesting systems, as magnetic saturation sources in magnetic sensors replacing electromagnets, or as magnetic scales in position measurement systems [2-5]. Furthermore, CoP alloys are also discussed as substitutes for conventional hard chromium coatings due to their high micro-hardnesses and corrosion resistances [6]. In the present work, the capability of electrochemical deposition is investigated for the fabrication of thick Co-based micromagnets, to be used as magnetic scales in a magnetic position sensing system.

Magnetic position measurement systems consist of a magnetic sensor reading the periodic magnetic stray field of a hard magnetic scale (Figure 1). Such systems are robust and cheap compared to their optical counterparts but do not achieve the same accuracy. A straightforward approach to reduce this accuracy gap is by shrinking the whole system including the magnetic scale, and the sensing structures, as well as the working distance. For this purpose, a CoP-based hard magnetic scale with a pitch of $100 \mu \mathrm{m}$ is developed by electrochemical deposition of hard magnetic $\mathrm{CoP}$ and $\mathrm{CoNiP}$. In combination with an existing tunnel magneto-resistive (TMR) sensor, an accuracy in the nanometer range is achieved [5]. The classic magnetic scale consists of alternating north and south poles written in magnetic tape. This opposes the approach presented here where the magnetic scale is an array of magnetic poles and nonmagnetic gaps uniformly magnetized in the out-of-plane direction.

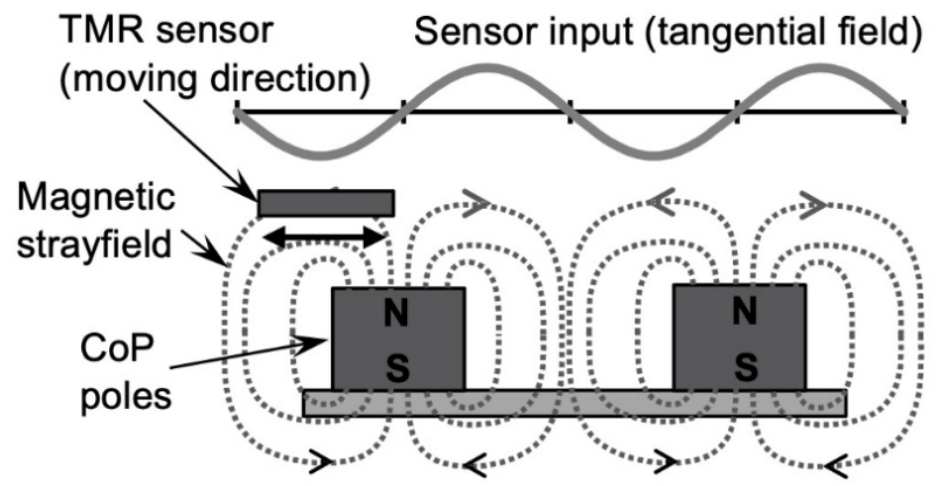

Figure 1. Schematic illustration of the sensor and scale arrangement. Sensor is sensitive to the tangential field and moves at a fixed working distance.

The hexagonal close-packed crystal structure of cobalt results in a strong intrinsic magnetic anisotropy and a high magnetic moment, which makes it a suitable material for the fabrication of hard magnets. Phosphorous, when alloyed with cobalt, is incorporated into the grain boundaries and increases coercivity by promoting the formation of single Co-grains by dendritic columnar growth [7-9]. Other elements that can be added are $\mathrm{Ni}, \mathrm{Pt}$ and Mn. The electrodeposited materials CoPtP and CoNiMnP are especially well-known for their high as-plated coercivity values and vertical magnetization directions $[5,7,10]$. CoPtP can furthermore be tempered at high temperatures to provide an ordered crystal structure, which improves the coercivity roughly ten-fold, [10] whilst often losing the IC compatibility. However, these materials possess two major disadvantages. One is the tradeoff of reducing the magnetic strength if a relatively high amount of an element like $\mathrm{P}$, $\mathrm{Mn}$ or Pt is incorporated. The other is the difficult process control, which hinders industrial use. Furthermore, for platinum, the content in the alloy is about $15 \%$. Since Pt is expensive, its use is not desirable for an economical industrial process. Binary CoP on the other hand can yield a high magnetic strength for a low $\mathrm{P}$ content in combination with better process control. The literature shows that even with this simple system, the direction of the magnetization can be tailored accordingly $[9,11-14]$. When a thick Co-based hard magnetic layer is micro-patterned, the shape anisotropy can be the dominant effect depending on the specific geometry. This shape anisotropy can easily overcome an out-of-plane crystal anisotropy, hence altering the final out-of-plane component of the magnetization in the 
application [11]. It is therefore mandatory to first develop and improve a process that yields a Co-based material with a high magnetic strength due to out-of-plane columnar growth, and a texture that exploits the crystal anisotropy. By doing this, the usable out-of-plane magnetization can be improved for the intended application. Another challenge with electrochemically-deposited compact $\mathrm{CoP}$ and $\mathrm{CoNiP}$ thick-films is to control the inherent mechanical strain so that crack-free micro-magnets with good adhesion can be produced.

Within the scope of this work, a process for the as-plated fabrication of a hard magnetic scale bar for a position measurement system was developed. Thereon, the correlation of electrodeposition process parameters to material properties is shown. The requirements for the material are a sufficient out-of-plane magnetization and coercivity, as well as a good geometrical fidelity. In the first step, the influence of the convection and current density on the deposition characteristics of $\mathrm{CoP}$ and $\mathrm{CoNiP}$ in a laboratory-scale setup was investigated. Then an integrated process for the fabrication of a hard magnetic scale bar with an out-of-plane magnetization was developed which qualifies as an industrial fabrication process. Furthermore, micro-hardness testing was carried out in order to investigate the elastic properties of the compact CoNiP layers.

\section{Materials and Methods}

\subsection{Electrochemical Deposition of Hard Magnetic CoP and CoNiP}

For the CoP deposition, a basic electrolyte according to [8] is used. Table 1 shows the composition of the electrolyte. The electrolyte contains cobalt (II) sulfate and sodium hypophosphite to provide the cobalt and phosphor ions. Boric acid acts as a $\mathrm{pH}$ buffer, and sodium sulfate increases the electrical conductivity of the electrolyte. A grain refiner/stress reliever (sodium saccharin) is essential to ensure an error-free plating with low residual stresses.

Table 1. Basic electrolyte.

\begin{tabular}{lc}
\hline \multicolumn{1}{c}{ Chemical } & $\begin{array}{c}\text { Concentration } \\
(\text { mol L }\end{array}$ \\
\hline Cobalt sulfate $\left(\mathrm{CoSO}_{4} 6 \mathrm{H}_{2} \mathrm{O}\right)$ & 0.12 \\
Sodium hypophosphite $\left(\mathrm{NaH}_{2} \mathrm{PO}_{2} 2 \mathrm{H}_{2} \mathrm{O}\right)$ & 0.06 \\
Boric acid $\left(\mathrm{H}_{3} \mathrm{BO}_{3}\right)$ & 0.49 \\
Sodium sulfate $\left(\mathrm{Na}_{2} \mathrm{SO}_{4}\right)$ & 0.16 \\
Sodium saccharin $\left(\mathrm{C}_{7} \mathrm{H}_{4} \mathrm{NO}_{3} \mathrm{SNa}_{2} \mathrm{H}_{2} \mathrm{O}\right)$ & 0.01 \\
\hline
\end{tabular}

The CoNiP electrolyte is identical except for the addition of $0.13\left(\mathrm{~mol} \mathrm{~L}^{-1}\right)$ nickel sulfate $\left(\mathrm{NiSO}_{4} \cdot 6 \mathrm{H}_{2} \mathrm{O}\right)$.

A modified rotating disc electrode (RDE) with a three-electrode setup is used to investigate the influences of mass transfer and current density on the deposition. The cathodes of the RDE experiments consist of a polished, high purity, copper disc embedded in an insulating cylinder. The rotation speed is varied between $n=0$ and $600 \mathrm{~min}^{-1}$. The deposition was carried out in galvanostatic mode with a constant current applied. The deposition time to reach a theoretical thickness of $10 \mu \mathrm{m}$ was calculated via Faraday's law assuming a current efficiency of $90 \%$. The exact thickness of each disc varies depending on the real current efficiency. This embedded copper disc with the deposited CoP and CoNiP layers is removed and magnetically characterized with a vibrating sample magnetometer (VSM). The discs have a diameter of $5 \mathrm{~mm}$ and thicknesses of 5 to $10 \mu \mathrm{m}$ resulting in a vanishing demagnetization factor if characterized with an in-plane ambient field. Hence it is possible to obtain the intrinsic magnetic parameters of the material without the disturbing influence of shape anisotropy. The magnetic volume of the samples is obtained by weighing, then converting the measured weight to a volume by utilizing the estimated density, which is based on the alloy composition as measured by EDX. The current efficiency is obtained by weighing the substrate before and after the deposition and comparing the measured value with the theoretical value derived from Faraday's law. 


\subsection{Integrated Fabrication of Hard Magnetic CoP Scales}

Based on the results from RDE experiments, first scales are fabricated in a $20 \mathrm{~L}$ electroplating cell with paddle agitation [12] and passive shielding to ensure stable forced convection conditions and a homogenous current density distribution. Since the CoP hard magnets were found to be less dependent on the convection conditions and exhibited a higher magnetic field, this material was chosen for the fabrication of the scales. The plating mask (resist) consists of a $55 \mu \mathrm{m}$ thick chemically amplified photoresist patterned by UV-lithography. The resist is very durable during plating conditions. Pre-patterned 4-inch silicon wafers with a $10 \mathrm{~nm}$ chromium adhesion layer and $200 \mathrm{~nm}$ of a copper seed layer are used as substrates. The scales are protected by a $300 \mathrm{~nm}$ silicon oxide-nitride-oxide $(\mathrm{ONO})$ stack and finally singularized by mechanical dicing.

For the magnetic characterization by means of a VSM, it was necessary to cut the scales in half to ensure reliable results due to sample size restrictions. The volume of a scale bar is estimated by weighing and surface profilometry.

Field-assisted deposition is used to study the induction of out-of-plane magnetic anisotropy. For this purpose, a homogeneous magnetic supporting field is applied perpendicular to the wafer plane (in-growth direction). This supporting field was generated by two ferrite permanent magnets $\left(10 \times 15 \times 2 \mathrm{~cm}^{3}\right)$. One of the magnets is placed directly behind the cathode with a $3 \mathrm{~mm}$ distance between the magnet surface and the wafer, and the other is placed behind the anode. The distance between anode and cathode is $16 \mathrm{~cm}$. By using this set-up, it is ensured that the CoP layers are exposed only to an out-of-plane magnetic field without meaningful in-plane components. By applying a homogeneous out-of-plane field with a magnetic field strength exceeding the coercivity, the spontaneous domain formation is hindered, and the material is deposited under the influence of a magnetic field. It must be considered that the strength of the magnetic field experienced inside the CoP body is reduced by the formation of a demagnetizing field. The magnetic field strength at the wafer surface was measured as $110 \mathrm{mT}(87 \mathrm{kA} / \mathrm{m})$ and thus exceeds the highest coercivity values of CoP obtained in this work.

\section{Results}

\subsection{Hard Magnetic CoP and CoNiP Layers}

The Co content for CoNiP in Figure 2 shows that by increasing the rotation speed more cobalt is incorporated at any given current density. For a lower rotation speed and higher current densities, Co is transported relatively slowly to the cathode surface compared to $\mathrm{Ni}$.

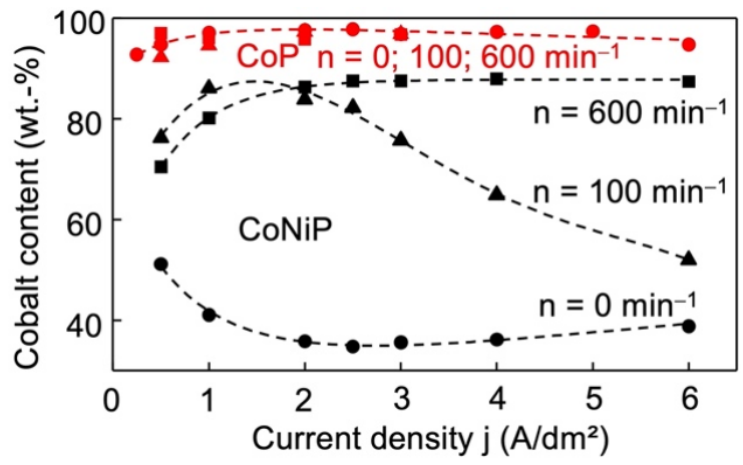

Figure 2. Dependency of the cobalt content on rotation speed (n) and current density. The investigations were carried out with the rotating disc electrode (RDE). The values are normalized for CoP and CoNiP, respectively.

The composition of ternary $\mathrm{CoNiP}$ is highly dependent on the current density and rotation speed as shown in Figure 2. A higher rotation speed results in a smaller diffusion layer in front of the cathode. It can be observed that the rate of cobalt reduction is affected by diffusion, since a rotation speed of $n=600 \mathrm{~min}^{-1}$ leads to a high Co content over the 
current density range, while deposition without forced convection $\left(n=0 \mathrm{~min}^{-1}\right)$ results in a reduced cobalt content, thus producing layers with poor magnetic properties. The composition of binary $\mathrm{CoP}$ on the other hand is less dependent on the mass transfer and current density, and therefore, it is easier to obtain layers with a defined composition.

Compact CoP and CoNiP layers with high current efficiencies of $>80 \%$ have been fabricated at $n=100$ and $600 \mathrm{~min}^{-1}$ in the current density ranges of 1 to $3 \mathrm{~A} / \mathrm{dm}^{2}$ for CoP and 2 to $3 \mathrm{~A} / \mathrm{dm}^{2}$ for CoNiP, respectively. Due to the mass-transfer controlled deposition of $\mathrm{CoNiP}$, there is no current density range in which the composition and the current efficiency are both stable and independent of the rotation speed of the RDE. At lower current densities and higher rotation speeds the current efficiency drops below 50\% (see Figure 3).

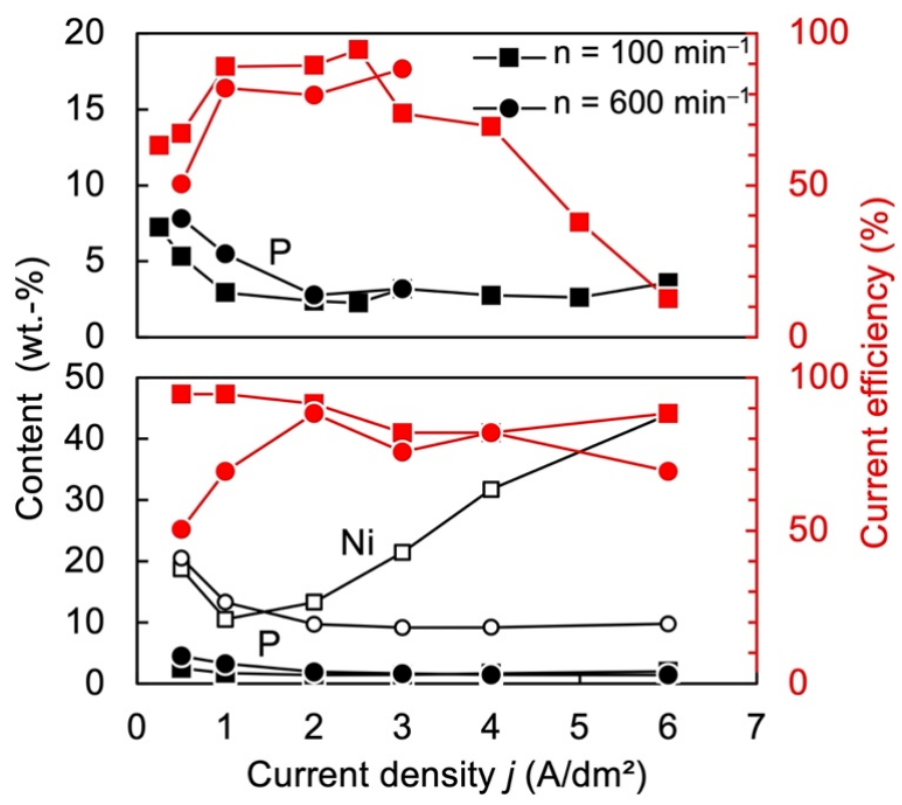

Figure 3. Dependency of the content of the non-cobalt elements on the current density together with the current efficiency for $\mathrm{CoP}$ (top) and $\mathrm{CoNiP}$ (bottom). The investigations were carried out with the rotating disc electrode (RDE). The element content is normalized to CoP and CoNiP, respectively.

Figure 4 shows that for $\mathrm{CoP}$ a magnetic strength of up to $0.7 \mathrm{~T}$ can be obtained in a narrow current density range around $2 \mathrm{~A} / \mathrm{dm}^{2}$. Although the $H_{C}$ and $J_{R}$ values for CoNiP remain steady over a wider current density range, it is less suitable for the designated application due to the generally lower remanence polarization (half of $\mathrm{CoP}$ ) and the strong influence of the convection, as indicated by a more pronounced shift to higher current densities for $n=600 \mathrm{~min}^{-1}$. Figures 3 and 4 show that the coercivity and the remanence polarization of $\mathrm{CoNiP}$ have an inverse relationship with the Ni content. It can be concluded that $\mathrm{CoP}$ yields much stronger magnets with reduced effort regarding process control. $\mathrm{CoNiP}$ on the other hand yields double the coercivity of CoP but the process requires precise control of the mass-transfer characteristics and the current density. Thus, regarding industrial production, $\mathrm{CoP}$ is the material of choice. 


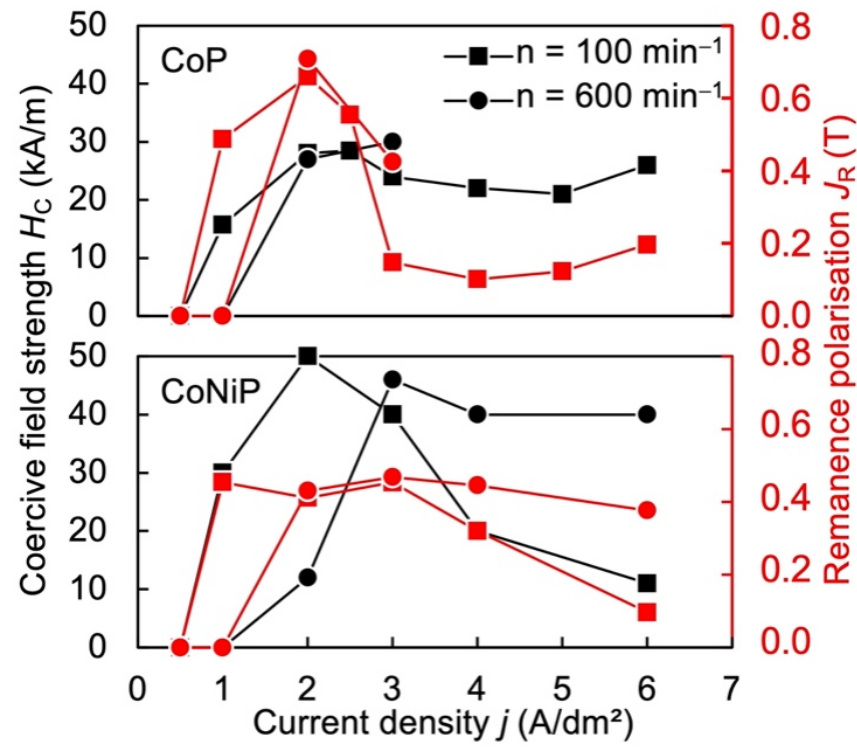

Figure 4. Correlation of current density to coercivity and remanence polarization for CoP (top) and CoNiP (bottom) at different rotation speeds (n). The investigations were carried out with the rotating disc electrode (RDE). The low coercivity values below $1 \mathrm{~A} / \mathrm{dm}^{2}$ are not reliable due to rotation of the magnetic moment during in-plane measurement.

As shown in Figure 5 it was possible to deposit $10 \mu \mathrm{m}$ thick compact CoP and CoNiP discs with intrinsic (in-plane) remanence polarizations of $J_{R}=0.65 \mathrm{~T}$ and $J_{R}=0.40 \mathrm{~T}$ and coercivities of $H_{C}=28 \mathrm{kA} / \mathrm{m}$ and $H_{C}=45 \mathrm{kA} / \mathrm{m}$, respectively.

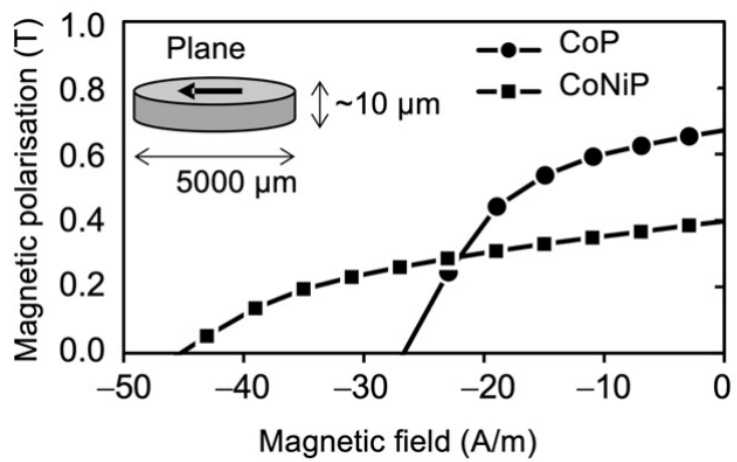

Figure 5. Example of an in-plane hysteresis curve for $\mathrm{CoP}$ and $\mathrm{CoNiP}$ for samples that show high current efficiency and a compact layer.

\subsection{Hard Magnetic CoP Scales}

A scanning electron microscopic (SEM) image of a CoP magnetic scale bar is shown in Figure 6. The scales were found to exhibit dominant in-plane shape anisotropy, which prevented the out-of-plane magnetization that is desired for the application (see Figure 1).

Figure 7 shows the two major components of the magnetization when an out-ofplane ambient magnetic field is applied. The VSM has a special coil set-up allowing the measurement of the magnetic moment in two (rectangular) in-plane directions [15]. From the figure, it can be seen that a large part of the magnetization remains in-plane due to the aforementioned pronounced shape anisotropy in that direction. 


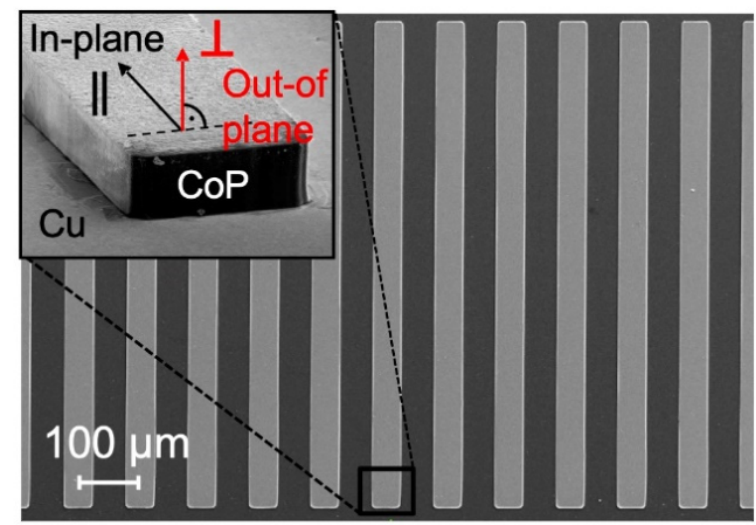

Figure 6. SEM top view of a CoP scale. The inlet indicates the in-plane and out-of-plane magnetization direction.

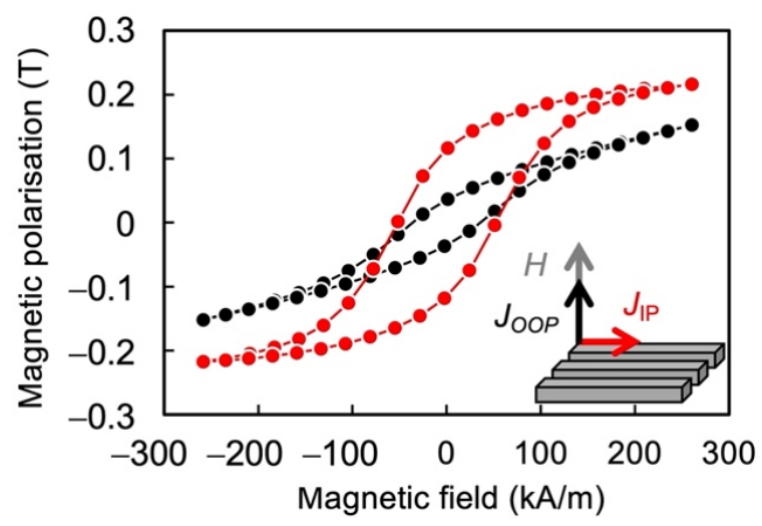

Figure 7. As-measured hysteresis loops of a CoP scale with the magnetic field applied in the out-ofplane direction showing the two major components of the magnetization.

To further improve the out-of-plane magnetization portion, geometrical variations have been investigated regarding their potential to induce vertical anisotropy. By increasing the thickness, the in-plane shape anisotropy can be reduced, thus improving the out-ofplane magnetization. Figure 8 shows that the out-of-plane loops have a lower remanence polarization JR compared to the in-plane loops as expected. The out-of-plane loops are smaller because the VSM used (maximum magnetic field of $260 \mathrm{kA} / \mathrm{m}$ ) is not capable of bringing the samples to a saturated state due to its high demagnetizing factor, especially for thin layers. The loops shown in Figure 8 are modified for the demagnetizing field by using an extrapolation method according to [16].

All loops in Figure 8 are measured at an identical external magnetic field of $200 \mathrm{kA} / \mathrm{m}$ in the VSM. Thus, the maximum magnetic polarization reached in the out-of-plane direction for the various sample thicknesses is extrapolated to the apparent maximum polarization of the in-plane measurement. This method only provides an estimated demagnetization factor, but the correction is still helpful in illustrating that the in-plane and out-of-plane measurements are hardly comparable because the samples experience a lower field in the out-of-plane direction, resulting in smaller loops being measured, which is in contrast to the external field that is needed in order to saturate thin films in the out-of-plane direction, and that can reach very high values. With a VSM, an out-of-plane remanence polarization of $170 \mathrm{mT}$ and a coercivity of $50 \mathrm{kA} / \mathrm{m}$ for the $55 \mu \mathrm{m}$ thick scales was obtained. The achievable plating thickness is limited due to the maximum photoresist thickness of the lithography process and the increasing residual stresses that lead to the formation of microcracks and adhesion losses. The $55 \mu \mathrm{m}$ thick scales exhibit micro-cracks and a rough surface topography (see Figure 8 bottom), both of which are undesirable for the application because of the quality of the tangential stray field portion (compare Figure 1), and thus the accuracy 
of the position measurement depends on the geometrical fidelity. The optimal thickness regarding substrate adhesion and geometrical fidelity is in the 25 to $30 \mu \mathrm{m}$ range.

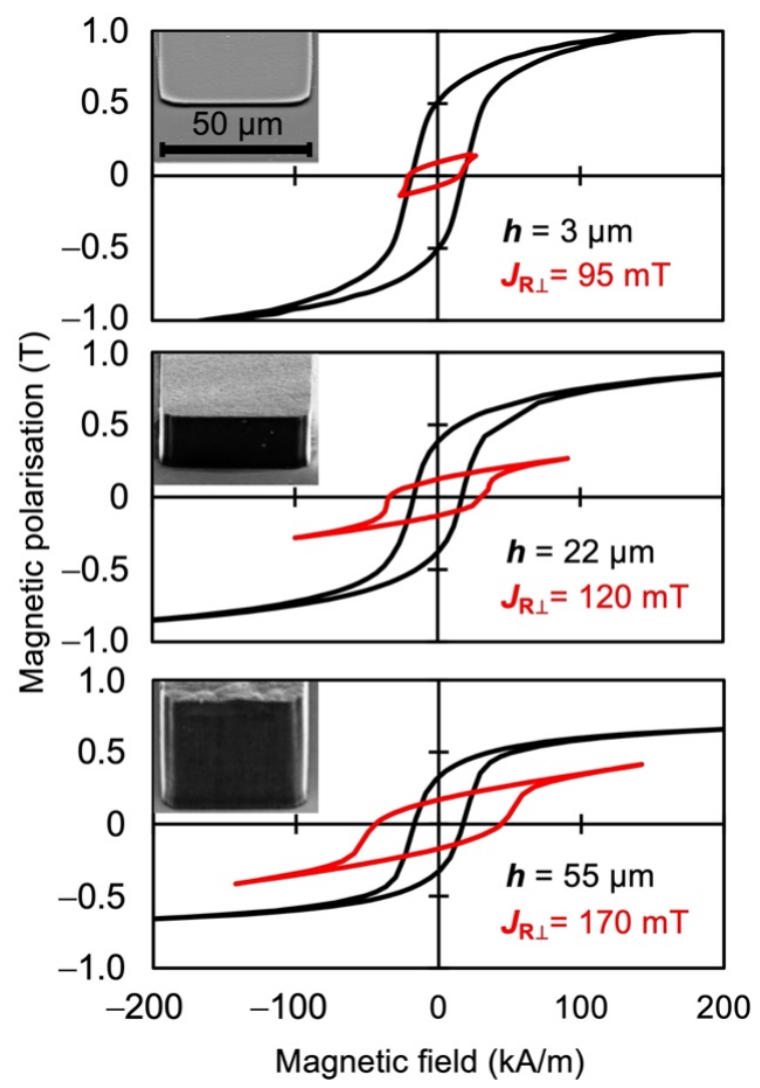

Figure 8. In-plane (black) and out-of-plane (blue) hysteresis loops of CoP scales with a thickness of $h=3,22$ and $55 \mu \mathrm{m}$. The in-plane and out-of-plane measurements are major and minor loops, respectively. The out-of-plane loops are calculated according to [16]. The scaling is identical in all three inlet pictures.

The obtained out-of-plane remanence polarizations of $170 \mathrm{mT}$ for the as-plated, and $205 \mathrm{mT}$ for the field-assisted depositions (Figure 9) exceed the $80 \mathrm{mT}$ and $190 \mathrm{mT}$ previously reported for the same material [13].

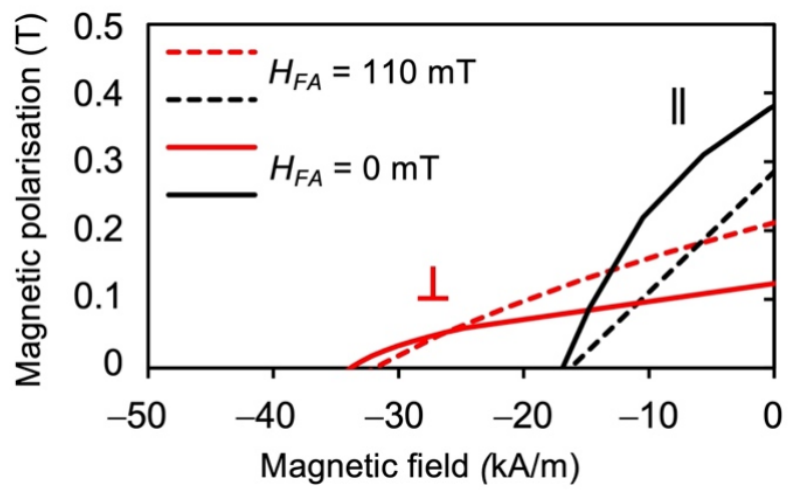

Figure 9. In-plane $(\|)$ and out-of-plane $(\perp)$ hysteresis loops of CoP scales deposited with and without an assisting field $\left(H_{F A}\right)$. 


\subsection{Assessment of the Magnetic Scale Operation}

To investigate the functionality of the magnetized magnetic scales, a test setup with a TMR position sensor was used. The sensor with a special design was positioned at a distance of about $30 \mu \mathrm{m}$ opposite to the magnetic scale on a high-resolution measurement bench as shown in Figure 10. The device employs a laser interferometer to provide a reference position signal, which is then compared to the position signal obtained by the sensor/scale system. With this test setup, the measurement bench was moved over the full-scale length $(25 \mathrm{~mm})$ while the position sensor signal was recorded. The absolute position error over the full scale was about $\pm 1 \mu \mathrm{m}$, which demonstrates the performance and usability of the CoP scales.

\section{Axis of movement}

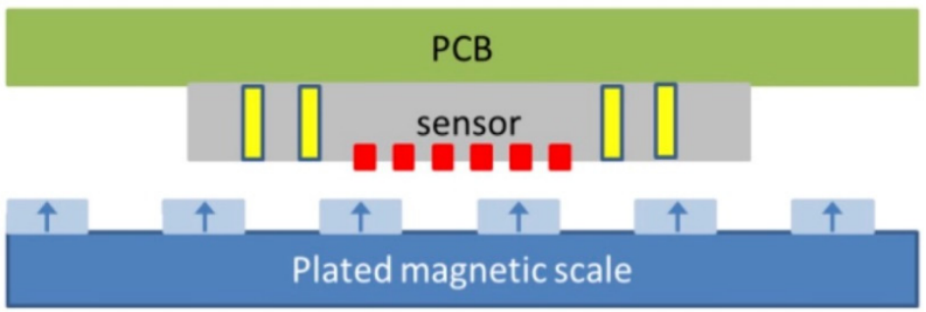

Figure 10. Setup of the measurement bench. The sensor chip is on top and the CoP scale is below.

\subsection{Further Planned Material Characterizations}

In a research project currently underway, the previous investigations are being further expanded, whereby the current results and assumptions are to be validated in more detail. The following section shows the first results for this, although they are not yet complete for all compositions. Rather, it is intended to show which additional analytical methods are to be used within the framework of the current research project in order to enable a comprehensive characterization of the deposited layers. In particular, the focus will be on the relationship between mechanical and magnetic properties.

The method of micro-hardness testing allows the characterization of thin layer systems deposited on substrates. Analysis of the load-displacement curve of the indentation test gives additional information regarding the elastic properties of the layer system because the measured elastic modulus from the load-displacement curve is related to the Youngs modulus. The samples B1, B2, B4 and B10 all had the same CoNiP- layer thickness of $10 \mu \mathrm{m}$. In Figure 11, the Vickers micro-hardnesses of four deposited CoNiP layers were determined using a Fisherscope HM 2000. The testing force used for the Vickers microhardness measurement series is $0.01961 \mathrm{~N}$ (HV0.002). The application time until reaching the specified testing force is $7 \mathrm{~s}$. The subsequent exposure time of the testing force is $14 \mathrm{~s}$. A total of ten individual measurements were performed on each deposited CoNiP layer. The typical indentation depth was $0.37 \mu \mathrm{m}$. The distance between the indentations is at least 6 times the average indentation diagonal length. Sample B1 was deposited with a current density $j$ of $2 \mathrm{~A} / \mathrm{dm}^{2}$. It shows a micro-hardness of approx. 583 to $795 \mathrm{HV} 0.002$. The elastic indentation modulus of sample B1 averages about 183.5 GPa. The micro-hardness of sample B2 also shows a higher scattering of 418 to $674 \mathrm{HV} 0.002$ (current density: $1 \mathrm{~A} / \mathrm{dm}^{2}$ ). The elastic indentation modulus, on the other hand, does not show any significant scatter. On average, this is about $200 \mathrm{GPa}$. The micro-hardness and the elastic indentation modulus of specimens B4 and B10 are comparable and show the lowest scatter within this comparison. The values range from 683 to 771 HV0.002 (micro-hardness), and with 185 GPa being calculated for the average elastic indentation modulus. Here, sample B4 was deposited with a current density of $1 \mathrm{~A} / \mathrm{dm}^{2}$ and B10 at $0.5 \mathrm{~A} / \mathrm{dm}^{2}$. The elastic indentation modulus shows a smaller range of variation in contrast to the micro-hardness series. The elastic indentation modulus is essentially determined by the atomic binding energy of the lattice, 
so the measured values are in the expected range (variations are rather small, compare Table 1).

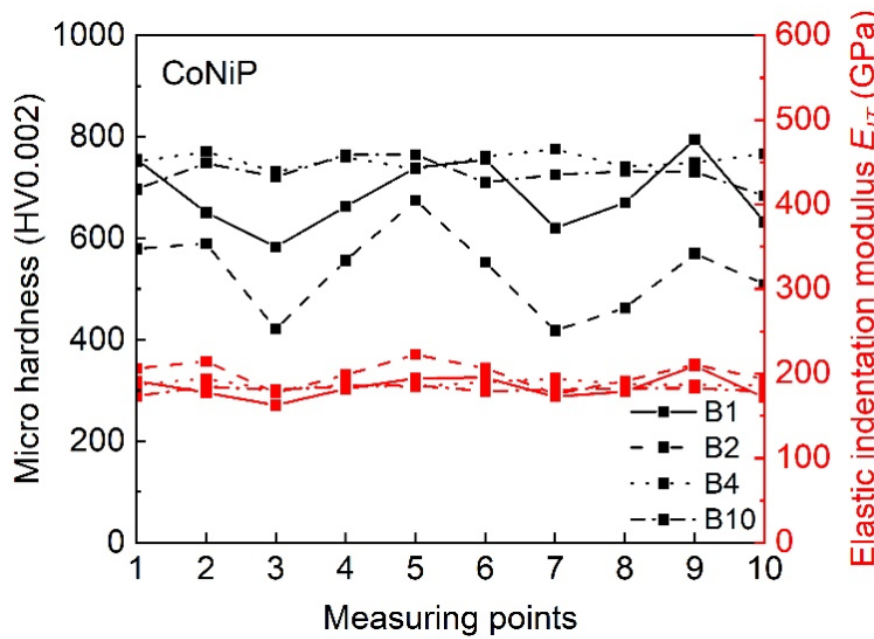

Figure 11. Micro-hardness and elastic Indentation Modulus $E_{I T}$ measurements for four deposited CoNiP samples.

Table 2 lists the mass percentages of the elements $\mathrm{Co}, \mathrm{Ni}$ and $\mathrm{P}$ in each of the deposited CoNiP samples (B1, B2, B4 and B10).

Table 2. Mass percentage of the elements $\mathrm{Co}, \mathrm{Ni}, \mathrm{P}$ in deposited CoNiP layers.

\begin{tabular}{cccc}
\hline & Co (Ma.-\%) & Ni (Ma.-\%) & P (Ma.-\%) \\
\hline B1 & 82.2 & 9.2 & 3.8 \\
B2 & 81.9 & 10.0 & 3.3 \\
B4 & 78.0 & 12.9 & 6.3 \\
B10 & 68.5 & 19.9 & 8.7 \\
\hline
\end{tabular}

By plotting the micro-hardness values of the samples (B1, B2, B4 and B10) vs. the phosphorus content (Figure 12), it can be seen that with an increasing phosphorus content the micro-hardness values become more homogeneous (also compare with Figure 11), and the average value increases. This behavior is in accordance with the results of other groups reported in the literature $[17,18]$.

CoP and CoNiP films offer the advantage that they can be deposited very homogeneously with respect to their microstructure. This is also evident with regard to their mechanical properties, such as hardness and indentation, and Young's modulus, respectively. With regard to hardness and elastic moduli, a dependence on the deposition temperature as well as the current density should be noted in particular, although this appears to have no influence on the homogeneity of the coatings [17,19]. In addition to these factors, the coating thickness, in particular, is of decisive importance, as it fundamentally influences not only the mechanical properties but also the magnetic properties of the coating [20]. These phenomena have also been confirmed in our own investigations. Due to the very good mechanical properties, which are characterized by high hardness and very good wear and corrosion resistance, CoP films, in particular, will be a good alternative to hard chrome coatings in the future, as already shown in Safavi et al. [6]. Environmentally safe process conditions can be used, allowing controlled deposition on various workpieces on a large scale, which is an important advantage over conventional hard chrome deposition. 


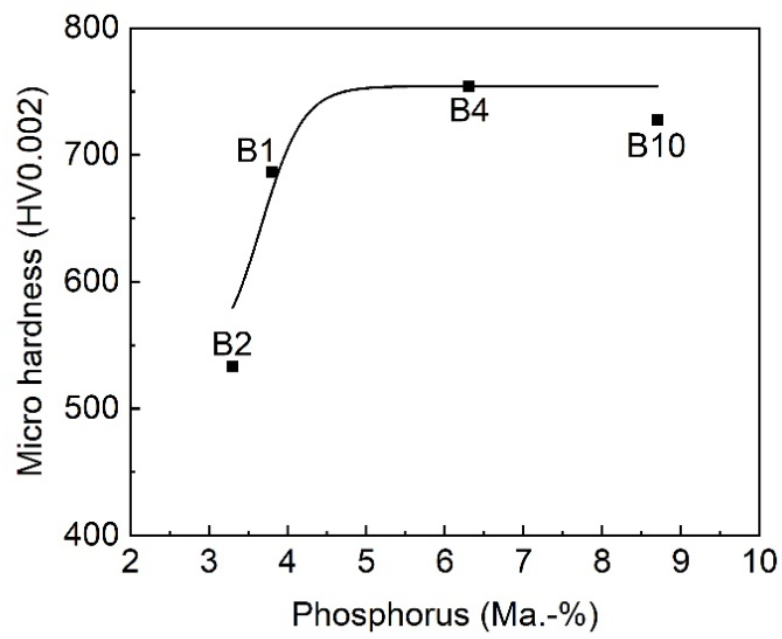

Figure 12. Average values of the micro-hardness of the deposited CoNiP layers in relation to the phosphorus content.

Figure 13 shows a transmission electron microscope (TEM) micrograph and an electron diffraction pattern of the cross-section of a CoP microscale. The TEM results show a rather nano-sized fibrous grain growth compared to the normally expected columnar growth associated with larger grains. This can be explained by the room temperature electroplating process and the use of a grain refinement additive, both of which help to suppress the mobility of surface atoms and thus promote the formation of smaller grains during electrodeposition.

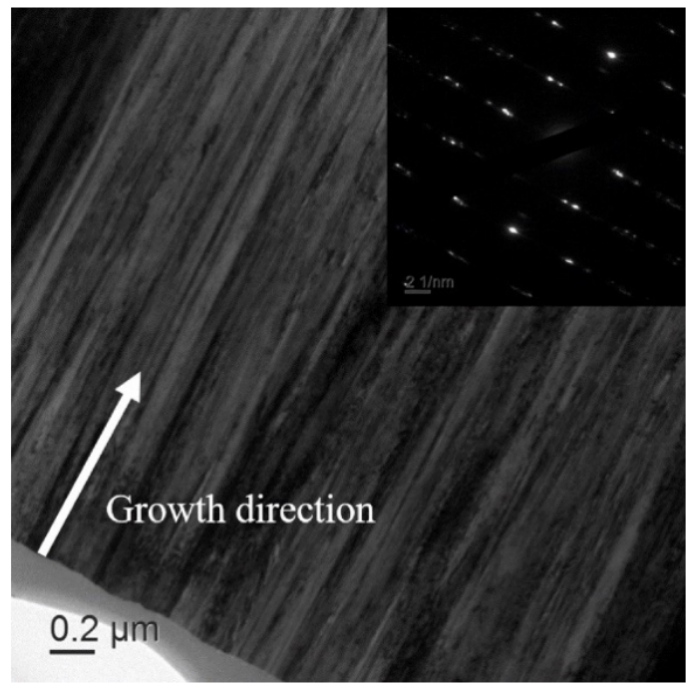

Figure 13. Cross-sectional TEM micrograph of electroplated CoP layers showing a preferred fibrous grain growth along the electrodeposition direction. The electron diffraction pattern (inlay) shows that the deposit consists of rather large crystals.

\section{Conclusions}

Within the scope of this study, a process for the fabrication of high moment hard magnetic CoP scale bars by means of electrochemical deposition was successfully developed and further improved. By carefully controlling the current density and the convection conditions it was possible to fabricate $\mathrm{CoP}$ and CoNiP layers with in-plane magnetic polarizations of $0.65 \mathrm{~T}$ and $0.40 \mathrm{~T}$ and in-plane coercivities of $28 \mathrm{kA} / \mathrm{m}$ and $45 \mathrm{kA} / \mathrm{m}$, respectively. CoP was picked as the material of choice for the scale bars due to its higher 
magnetic strength values, which are desirable for their application to ensure a strong enough TMR sensor signal for working distances ranging from 20 to $90 \mu \mathrm{m}$. The optimum thickness, which yields CoP scale bars with good adhesion, no micro-cracks, and a smooth surface morphology is found to be in the $25-30 \mu \mathrm{m}$ range. Thicker layers of up to $55 \mu \mathrm{m}$ develop a coarse surface morphology with micro-cracks which can alter the tangential stray field thus hindering the accuracy of the position measurement in the final application. By improving the electroplating process of $\mathrm{CoP}$ in order to achieve maximum magnetic strength and a strong fibrous out-of-plane grain growth it was possible to partially overcome the obstructive in-plane shape anisotropy, thus fabricating magnetic scales with a sufficient out-of-plane magnetization for the application. The obtained out-of-plane remanence polarizations of $170 \mathrm{mT}$ for the as-plated despositions and $205 \mathrm{mT}$ for the field-assisted depositions exceed the $80 \mathrm{mT}$ and $190 \mathrm{mT}$ previously reported for the same material. Moreover, the binary CoP process imposes lower requirements regarding process control compared to the ternary CoNiP system and is, therefore, more suitable for an industrial application. Due to the aforementioned advantages, binary CoP shows a sufficient out-ofplane magnetization for the use as micro-structured magnetic scale bars.

In a first approach, mechanical quantities in terms of micro-hardness were measured for selected samples, which will be used in the future to correlate with the magnetic properties. These investigations are currently in the early stages and are only intended to provide an outlook on the future research strategy at this moment.

Author Contributions: Conceptualization, M.T. and M.S.; methodology, M.T. and M.S.; validation, H.K., investigation, M.T., T.B., P.S. and H.K.; data curation, M.T. and M.S.; writing-original draft preparation, M.T., M.S., T.B. and P.S.; writing-review and editing, M.T., M.S., T.B., P.S. and H.K.; supervision, M.S.; funding acquisition, M.S. All authors have read and agreed to the published version of the manuscript.

Funding: This research was funded by the German Federal Ministry of Education and Research (BMBF), grant number 16ES0191.

Institutional Review Board Statement: Not applicable.

Informed Consent Statement: Not applicable.

Data Availability Statement: The data presented in this study are available on request from the corresponding author.

Acknowledgments: The authors gratefully acknowledge Detlev Cassel, Petra Böswald, and Heike Müller, as well as Rainer Lilischkis for technical support and EDX measurements, respectively. The authors would like to thank all project partners involved. We especially thank the German Federal Ministry of Education and Research (BMBF) for funding this project under the grant ID 16ES0191.

Conflicts of Interest: The authors declare no conflict of interest.

\section{References}

1. Sharma, S.; Radulov, I.; Major, M.; Alff, L. Evolution of Magnetic Anisotropy with Sm Contents in Sm-Co Thin Films. IEEE Trans. Magn. 2018, 54, 11. [CrossRef]

2. Reimer, T.; Lofink, F.; Lisec, T.; Thede, C.; Chemnitz, S.; Wagner, B. Temperature-stable NdFeB micromagnets with high-energy density compatible with CMOS back end of line technology. MRS Adv. 2016, 1, 209-213. [CrossRef]

3. Arnold, D.P.; Wang, N. Permanent Magnets for MEMS. J. Micromechanical Syst. 2009, 18, 1255-1266. [CrossRef]

4. $\quad$ Myung, N.V.; Park, D.-Y.; Yoo, B.-Y.; Sumodjo, P.T.A. Development of electroplated magnetic materials for MEMS. J. Magn. Magn. Mater. 2003, 265, 189-198. [CrossRef]

5. Paul, J.; Knoll, H.; Lenkl, A.; Tide, R.; Theis, M.; Saumer, M.; Vetter, C.; Piorra, A.; Meyners, D.; Lofink, F.; et al. Hochauflösende Magnetfeldpositionssensoren zur Präzisen Steuerung von Produktionsanlagen. In Proceedings of the MikroSystemTechnikKongress, Karlsruhe, Germany, 26-28 October 2015.

6. Safavi, S.; Walsh, F.C. Elextrodeposited Co-P alloy and composite coatings: A review of progress towards replacement of conventional hard chomic deposits. Surf. Coat. Technol. 2021, 422, 127564. [CrossRef]

7. Winterstein, V. Magnetische Eigenschaften und Modellierung des Ummagnestisierungsverhaltens Galvanisch Abgeschiedener Anisotroper Kobalt Speicherschichten, 2nd ed.; Verlag ISLE: Illmenau, Germany, 1997. 
8. Guan, S.; Nelson, B.J. Pulse-Reverse Electrodeposited Nanograinsized CoNiP Thin Films and Microarrays for MEMS Actuators. J. Electrochem. Soc. 2005, 152, 190-195. [CrossRef]

9. Armyanov, S. Crystallographic structure and magnetic properties of electrodeposited cobalt and cobalt alloys. Electrochim. Acta 2000, 45, 3323-3335. [CrossRef]

10. Kulkarni, S.; Roy, S. Deposition of thick Co-rich CoPtP films with high energy product for magnetic microelectromechanical applications. J. Magn. Magn. Mater. 2010, 322, 1592-1596. [CrossRef]

11. Oniku, O.D.; Qi, B.; Arnold, D.P. Electroplated thick-film cobalt platinum permanent magnets. J. Magn. Magn. Mater. 2016, 416, 417-428. [CrossRef]

12. Delbos, S.; Chassaing, E.; Grand, P.P.; Weitbrecht, V.; Bleninger, T. Controlled Mixing and Transport in Comb-Like and Random Jet Array Stirring Systems, in Advanced Topics in Mass Transfer; El-Amin, M., Ed.; IntechOpen: London, UK, 2011. [CrossRef]

13. Cho, H.J.C.; Bhansali, S.; Ahn, C.H. Electroplated thick permanent magnet arrays with controlled direction of magnetization for MEMS application. J. Appl. Phys. 2000, 87, 6340-6342. [CrossRef]

14. Hsiao, C.-J.; Hsiao, H.-S.; Tseng, C.-Y.; Chang, J.-Y.; Sung, C.-K.; Wang, S.-C.; Chin, T.-S. Enhancement in (002) texture of electroplated Co-based hard magnet layers. AIP Adv. 2017, 7, 0562102-0562106. [CrossRef]

15. Samwel, E.O. Magnetic Characterisation of Recording Materials: Design, Instrumentation and Experimental Method. Ph.D. Thesis, University Twente, Enschede, The Netherlands, 1995.

16. Dobrynin, A.; Dempsey, N. Experimental determination of the magnetization dependent part of the demagnetizing field in hard magnetic materials. Appl. Phys. Lett. 2010, 97, 1925061-1925063. [CrossRef]

17. Cojocaru, P.; Magagnin, L.; Gómez, E.; Vallés, E. Electrodeposition of CoNi and CoNiP alloys in sulphamate electrolytes. J. Alloy. Compd. 2010, 503, 454-459. [CrossRef]

18. Emerson, R.N.; Kennady, C.J.; Ganesan, S. Effect of Phosphorous Source Material on the Electrodeposition of CoNiP Magnetic Films. J. Appl. Sci. 2006, 6, 227-232. [CrossRef]

19. Mirzamaani, M.; Romankiw, L.; McGrath, C.; Karasinski, J.; Mahlke, J.; Anderson, N.C. Correlation of structure and magnetic properties of thin CoP films. J. Electrochem. Soc. 1988, 135, 2813. [CrossRef]

20. Ciudad, D.; Prieto, J.L.; Lucas, I.; Aroca, C.; Sanchez, P. Optimization of magnetic properties of electrodeposited CoP multilayers for sensor applications. J. Appl. Phys. 2007, 101, 043907. [CrossRef] 